\title{
The Application of Tirofiban in the Endovascular Treatment of Acute Ischemic Stroke: A Meta-Analysis
}

\author{
Lisha Tang Xiangqi Tang Qianwen Yang \\ Department of Neurology, The Second Xiangya Hospital, Central South University, Changsha, China
}

\section{Keywords}

Tirofiban · Acute ischemic stroke · Endovascular treatment

\begin{abstract}
Objective: The purpose of this meta-analysis is to evaluate the safety and efficacy of tirofiban during endovascular treatment (EVT) for acute ischemic stroke (AIS) patients. Methods: We systematically searched PubMed, Embase, Web of Science, and CENTRAL (Cochrane Central Register of Controlled Trials) databases for randomized controlled trials and cohort studies (published before May 1, 2020; no language restrictions) comparing tirofiban administration to blank control during EVT in patients with AIS. Our primary end points were the 3-month functional outcome, recanalization rate, symptomatic intracerebral hemorrhage, and 3-month mortality. Results: The incidence of 3-month modified Rankin Scale (mRS) 0-2 score of the tirofiban group was higher than that of the control group (odds ratio $[\mathrm{OR}]=1.27$, $95 \% \mathrm{Cl}[1.09,1.48], p=0.002)$ with heterogeneity $\left(l^{2}=34 \%\right.$, $p=0.11$ ). Data pooled from the 6 studies describing the details of retriever stent in EVT revealed that tirofiban was associated with higher incidence of 3-month mRS 0-2 score $(\mathrm{OR}=1.48,95 \% \mathrm{Cl}[1.11,1.96], p=0.007)$. The recanalization rate was higher in the tirofiban group compared to the control group $(\mathrm{OR}=1.66,95 \% \mathrm{Cl}[1.16,2.39], p=0.006)$. There
\end{abstract}

were no statistically significant differences in the incidence of symptomatic intracranial hemorrhage $(\mathrm{OR}=0.97,95 \% \mathrm{Cl}$ $[0.73,1.31], p=0.86)$ and intracranial hemorrhage $(\mathrm{OR}=1.08$, $95 \% \mathrm{Cl}[0.59,1.97], p=0.80$ ) between tirofiban and non-tirofiban group. Besides, the tirofiban administration was associated with lower mortality ( $\mathrm{OR}=0.75,95 \% \mathrm{Cl}[0.62,0.91]$, $p=0.003)$. Conclusions: The application of tirofiban in EVT of AIS may improve functional outcomes and reduce mortality at 3 months. Besides, tirofiban does not seem to increase the risk of symptomatic intracranial hemorrhage and intracranial hemorrhage, either in the anterior or posterior circulation stroke.

(c) 2021 S. Karger AG, Basel

\section{Introduction}

Ischemic stroke is the most common cerebrovascular accident caused by interruption of cerebral blood flow, accounting for about $87 \%$ of all strokes [1]. Ischemic stroke seriously threatens human health and has the characteristics of high morbidity, high disability, and high mortality. The only effective treatment for acute ischemic stroke (AIS) is to timely restore sufficient blood perfusion and efficiently salvage the ischemic penumbra. Intravenous thrombolysis within 3-4.5 h after onset is the pre-

$\begin{aligned} & \text { karger@karger.com } \\ & \text { www.karger.com/ced }\end{aligned}$
Karger ${ }^{\prime /}$


ferred method for the treatment of AIS in the super acute phase [2]. But the limited therapeutic time window and complex contraindications limit its application. Clinically, AIS patients treated with intravenous alteplase only accounted for a small part of the total.

In recent years, endovascular treatment (EVT) technology has been gradually but steadily improved, providing opportunities to expand the treatment window and improve revascularization rates. There are several EVT approaches, including mechanical thrombectomy, angioplasty, and stenting, and each approach has its own targeted AIS patients. From 2015 to 2016, the results of 7 large-scale randomized controlled trials [3-9] showed that EVT could increase the recanalization rate of patients with AIS in anterior circulation arterial occlusion and significantly improve long-term prognosis. But at the same time, endovascular operation procedure may cause endothelial damage, platelet activation, and subsequent early reocclusion [10-12].

Tirofiban is a highly selective, reversible, non-peptide platelet glycoprotein (GP) IIb/IIIa receptor antagonist, blocking the final pathway of platelet aggregation and subsequent thrombus formation. Tirofiban has been approved by the US Food and Drug Administration for the treatment of acute coronary syndrome [13] but not yet for AIS. Previous studies have confirmed that intravenous tirofiban administration is safe in patients with AIS $[14,15]$, but clinical studies on the efficacy and safety of tirofiban in the EVT of AIS have no consistent conclusions $[16,17]$. The use of tirofiban in cerebrovascular therapy has not been evaluated as rigorously as in coronary intervention. Here, we conducted a meta-analysis to assess the safety and efficacy of tirofiban in the treatment of EVT for AIS.

\section{Materials and Methods}

\section{Search Strategy}

This meta-analysis is reported in accordance with the PRISMA guidelines. We systematically searched PubMed, Embase, Web of Science, and CENTRAL (Cochrane Central Register of Controlled Trials) databases for randomized controlled trials and cohort studies (published before May 1, 2020; no language restrictions) comparing tirofiban administration to blank control during EVT in patients with AIS. Two researchers independently conducted the literature search. We used a combination of following Mesh and free terms: AIS, brain/cerebral infarction, stroke, cerebrovascular disease, cerebral ischemia, cerebral embolism, tirofiban, GP IIb/IIIa receptor antagonist, EVT, angioplasty, mechanical thrombectomy, and stenting. The reference lists of eligible studies and relevant reviews were manually reviewed for potential inclusion.

\section{Selection Criteria}

The inclusion criteria were (1) clinical diagnosis of AIS; (2) computed tomography scan excluded intracranial hemorrhage; (3) occlusion of large vessels confirmed by imaging examination (DSA or CTA); (4) receiving EVT within the time window; and (5) treated with tirofiban based on conventional treatment. The exclusion criteria were (1) studies with ambiguous intervention measures; (2) studies with an unclear description of grouping or testing; (3) uncompleted clinical trial; and (4) letters, review, correspondence, conference abstracts, descriptive studies, and animal experiment.

\section{Data Extraction and Quality Assessment}

Data for each eligible study were extracted by 2 authors independently. Any disagreements were resolved by discussion. The following details were sourced: authors, publication year, study design, demographic information, tirofiban protocol, and outcomes. The efficacy outcomes were the 3-month modified Rankin Scale (mRS) score $0-2$, and recanalization rate. Artery recanalization was assessed according to thrombolysis in Cerebral Infarction (TICI) scale, with successful recanalization defined as TICI $\geq 2 b$. The safety outcomes were the incidence of symptomatic intracerebral hemorrhage (sICH) and intracerebral hemorrhage (ICH) and mortality at 90 days. sICH was defined according to the ECASS III definition [18] and death was an mRS score of 6 . The Cochrane Risk of Bias Tool was used to assess the quality of RCTs. The quality of observational studies was assessed using the Newcastle-Ottawa Scale. In general, the RCT was of high methodological quality, and entire observational studies were of high quality with satisfactory scores.

\section{Statistical Analysis}

Statistical analyses were performed using Review Manager (v.5.3) with statistical significance defined as a $p$ value of $<0.05$ if not explicitly stated otherwise. We calculated pooled estimates of the odds ratio $(\mathrm{OR})$ for categorical variables and mean difference for continuous variables with random-effects model. Heterogeneity was assessed using the Cochran $Q$ test and $I^{2}$ test. The fixedeffects model was used if $I^{2}<50 \%$ (defined as low heterogeneity); otherwise, the random-effects model was applied. A sensitivity analysis was performed to assess the influence of each study on the overall results by omitting studies, in turn, one by one. Publication bias was evaluated via funnel plot analysis. We assessed the symmetry using Begg and Egger tests and defined significant publication bias as a $p$ value $<0.1$. Publication bias was evaluated with Stata (v.12).

\section{Results}

\section{Study Selection and Characteristics of Eligible Studies}

The selection process is shown in Figure 1. A total of 966 potentially relevant studies were identified in the database search. After the exclusion of 165 duplicated studies, 801 studies remained. After a title and abstract screen, 772 studies were excluded. Of 29 full texts retrievable for detailed evaluation, 15 articles were further excluded be- 


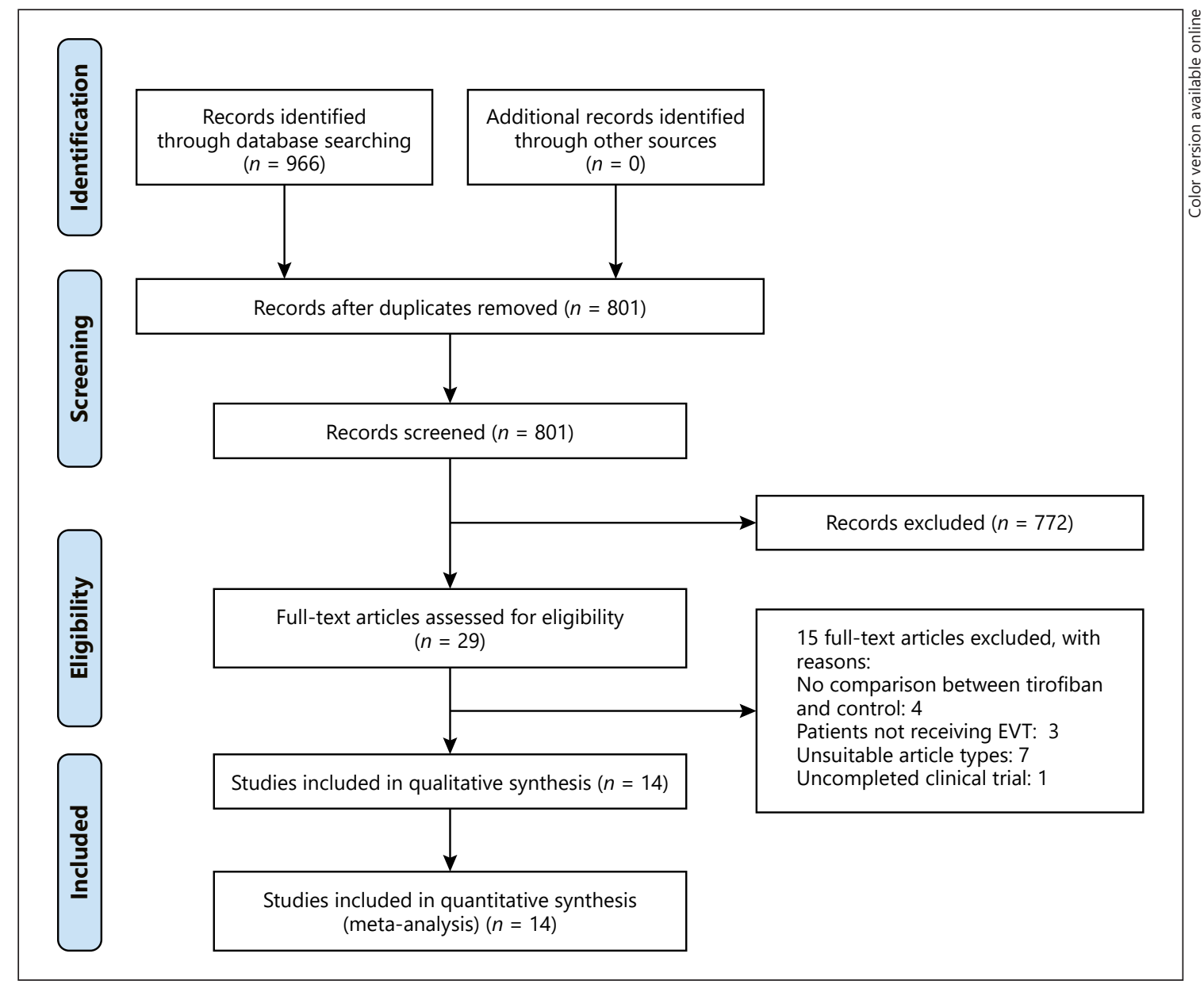

Fig. 1. Flow diagram of the study selection procedure. EVT, endovascular treatment.

cause of no comparison between tirofiban and control, not receiving EVT, unsuitable article types, and uncompleted clinical trials. Ultimately, 14 studies eligible were included in our meta-analysis, and the basic characteristics of the included studies are shown in Table 1.

\section{The Efficacy of Tirofiban}

Data on the occurrence of 3-month mRS 0-2 score were available in 14 studies [16,17, 19-30] involving a total of 3,251 patients (1,169 with tirofiban and 2,082 without tirofiban). The incidence of 3-month mRS 0-2 score of the tirofiban group was higher than that of the control group $(\mathrm{OR}=1.27,95 \% \mathrm{Cl}[1.09,1.48], p=0.002$; shown in Fig. 2) with heterogeneity $\left(I^{2}=34 \%, p=0.11\right)$. There were discrepancies in the subgroup analysis results. Subjects of anterior circulation stroke (ACS) and posterior circulation stroke (PCS) were analyzed, respectively.
In the $\mathrm{ACS}(\mathrm{OR}=1.11,95 \% \mathrm{Cl}[0.88,1.39], p=0.38)$ and PCS $(\mathrm{OR}=1.11,95 \% \mathrm{Cl}[0.75,1.63], p=0.61)$ subgroups, the incidences of 3-month mRS 0-2 score were not significantly different between subjects treated with tirofiban and those not receiving it (shown in Fig. 3). Of the available studies, only 6 studies [16, 20, 21, 24, 25, 27] described the details of the specific operation process in EVT, and all of them used retriever stent. The data pooled from the 6 studies involving 350 patients in the tirofiban group and 625 patients in the non-tirofiban group revealed that tirofiban was associated with a higher incidence of 3-month mRS $0-2$ score $(\mathrm{OR}=1.48,95 \% \mathrm{Cl}$ $[1.11,1.96], p=0.007$; shown in Fig. 4). The analysis was homogenous $\left(I^{2}=0 \%, p=0.68\right)$. Five studies $[20,21,25$, $27,29]$ reported the recanalization rate in 1,262 patients with low heterogeneity $\left(p=0.27, I^{2}=23 \%\right)$. The recanalization rate was higher in the tirofiban group compared 


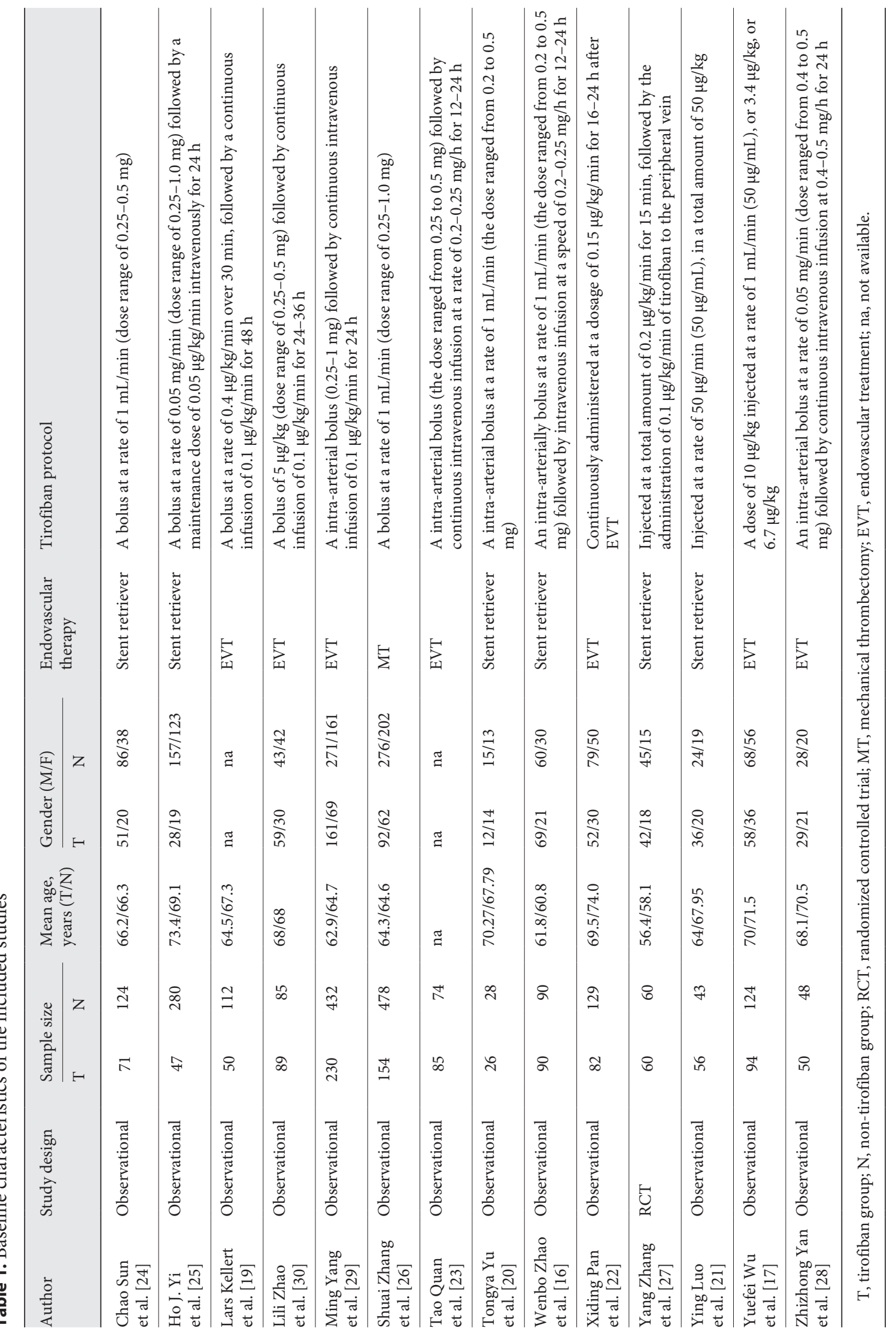




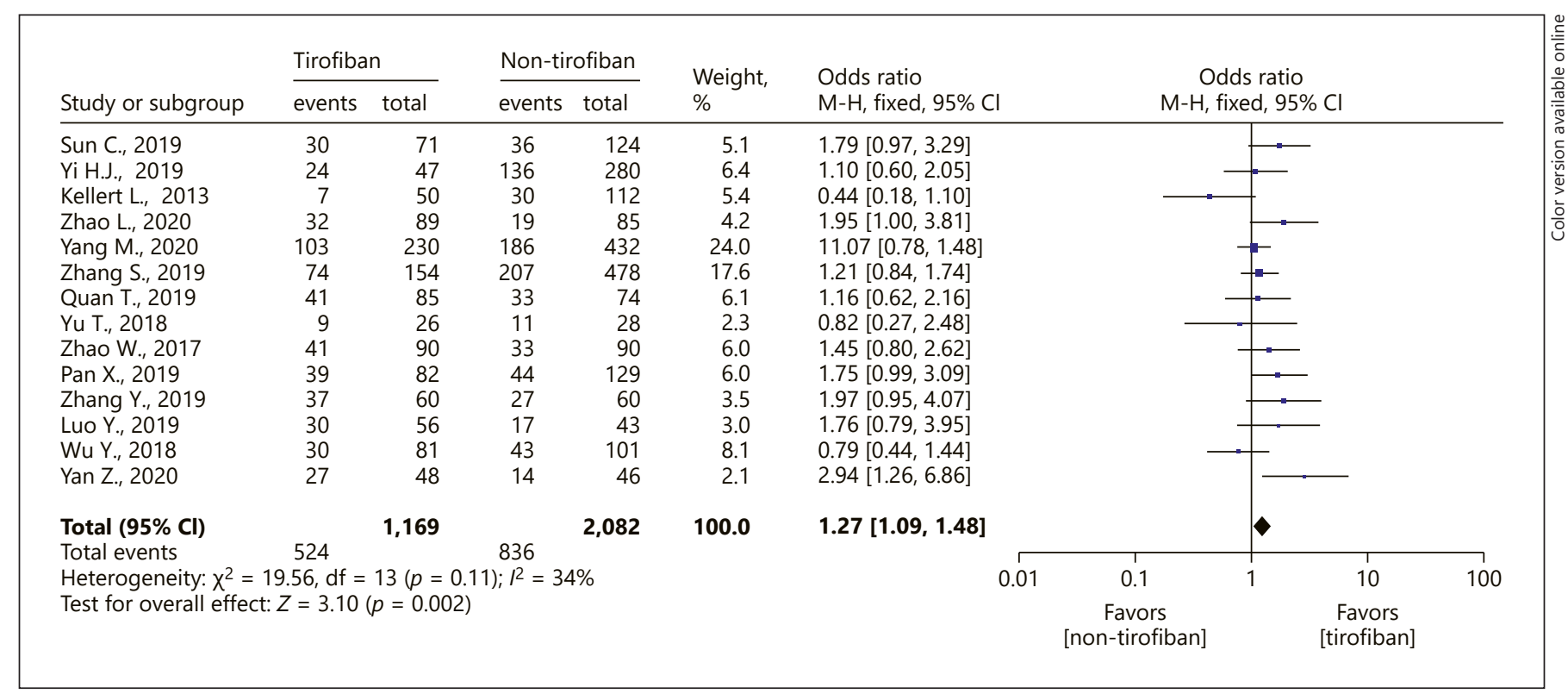

Fig. 2. Forest plot and meta-analysis of the incidence of 3-month mRS 0-2 score. mRS, modified Rankin Scale.

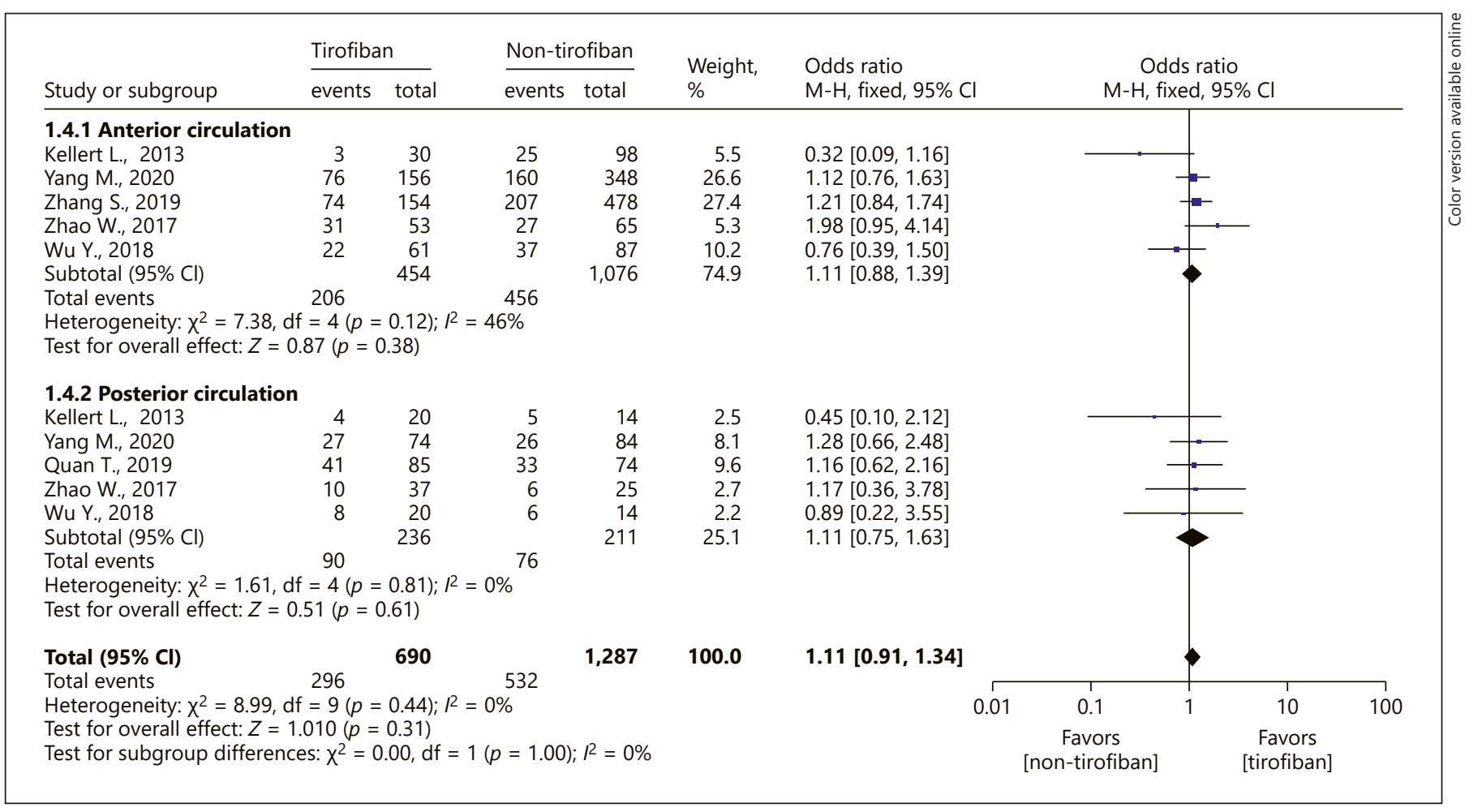

Fig. 3. Forest plot and meta-analysis of subgroup analysis of 3-month mRS 0-2 score in the anterior and posterior circulation. mRS, modified Rankin Scale. 


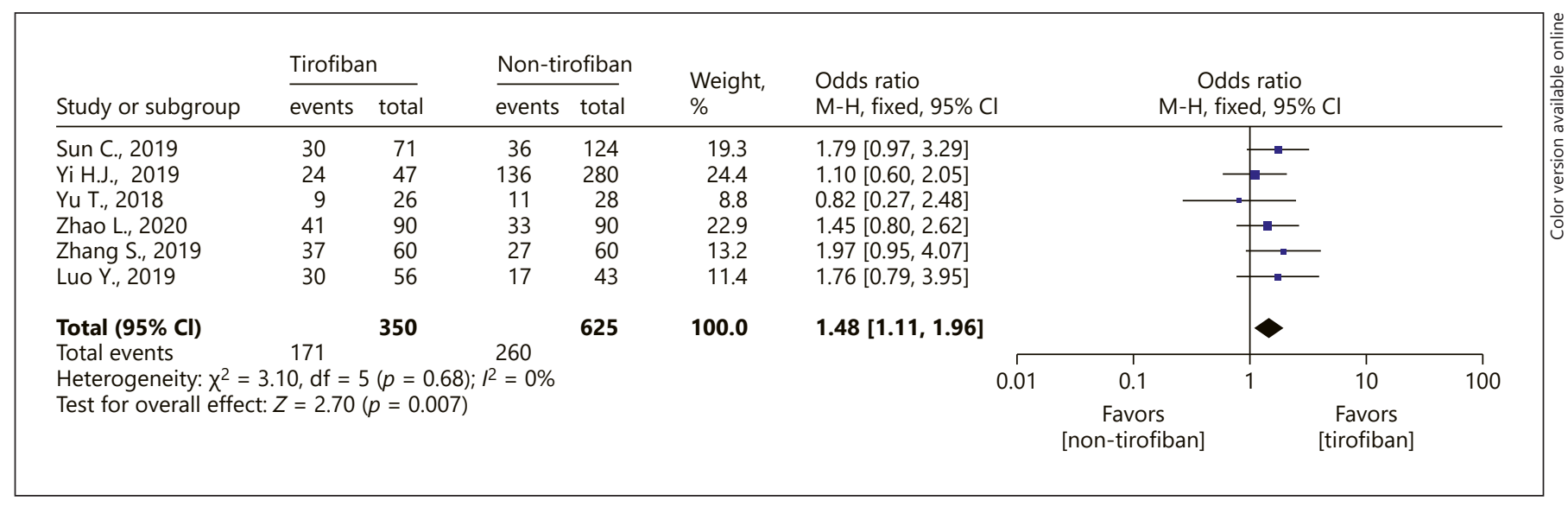

Fig. 4. Forest plot and meta-analysis of the incidence of 3-month mRS 0-2 score in retriever stent subgroup. mRS, modified Rankin Scale.

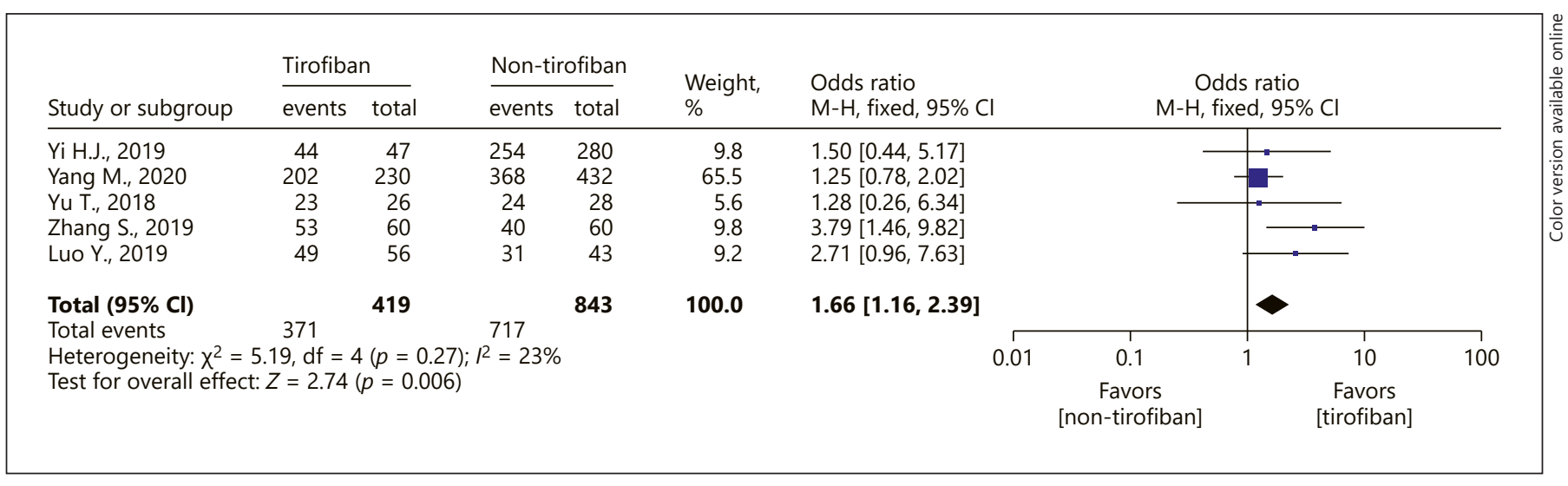

Fig. 5. Forest plot and meta-analysis of postoperative recanalization rate.

to the control group (OR $=1.66,95 \% \mathrm{CI}[1.16,2.39], p=$ 0.006; shown in Fig. 5).

\section{The Safety of Tirofiban}

sICH data were available for 2,450 patients from 12 studies [16, 17, 19-28]. The incidence of sICH was $9.42 \%$ $(81 / 860)$ in the tirofiban group and $10.38 \%(165 / 1,590)$ in the non-tirofiban group. The difference was not statistically significant $(\mathrm{OR}=0.97,95 \% \mathrm{CI}[0.73,1.31], p=0.86$; $I^{2}=23 \%$; shown in Fig. 6). The incidences of sICH were consistent in the subgroups of ACS and PCS. Six studies $[16,17,19,23,26,29]$ evaluated postoperative sICH in the anterior or posterior circulation. Pooled analysis indicated no significant difference between the tirofiban group and non-tirofiban group in ACS (OR $=1.38,95 \% \mathrm{CI}$
$[0.78,2.41], p=0.27$; heterogeneity: $\left.I^{2}=56 \%, p=0.06\right)$ or PCS (OR $=0.94,95 \%$ CI $[0.34,2.62], p=0.91$; heterogeneity: $I^{2}=0 \%, p=0.47$ ), which is shown in Figure 7 . In patients treated with retriever stent, sICH was reported in 25 out of 350 patients $(7.14 \%)$ receiving tirofiban and in 45 out of 625 patients (7.20\%) not receiving the drug. The difference was not statistically significant $(\mathrm{OR}=0.86,95 \%$ CI $[0.50,1.48], p=0.59 ; I^{2}=0 \%$; shown in Fig. 8 ).

Pooled analysis of the 6 studies [16, 17, 24, 26, 27, 29] that assessed the ICH showed that there was no significant difference in occurrence of ICH between the tirofiban group and non-tirofiban group (OR $=1.08,95 \% \mathrm{CI}$ $[0.59,1.97], p=0.80$; shown in Fig. 9) with statistically significant between-study heterogeneity $\left(I^{2}=84 \%\right.$, $p<$ $0.00001)$. Sensitivity analysis showed that heterogeneity 


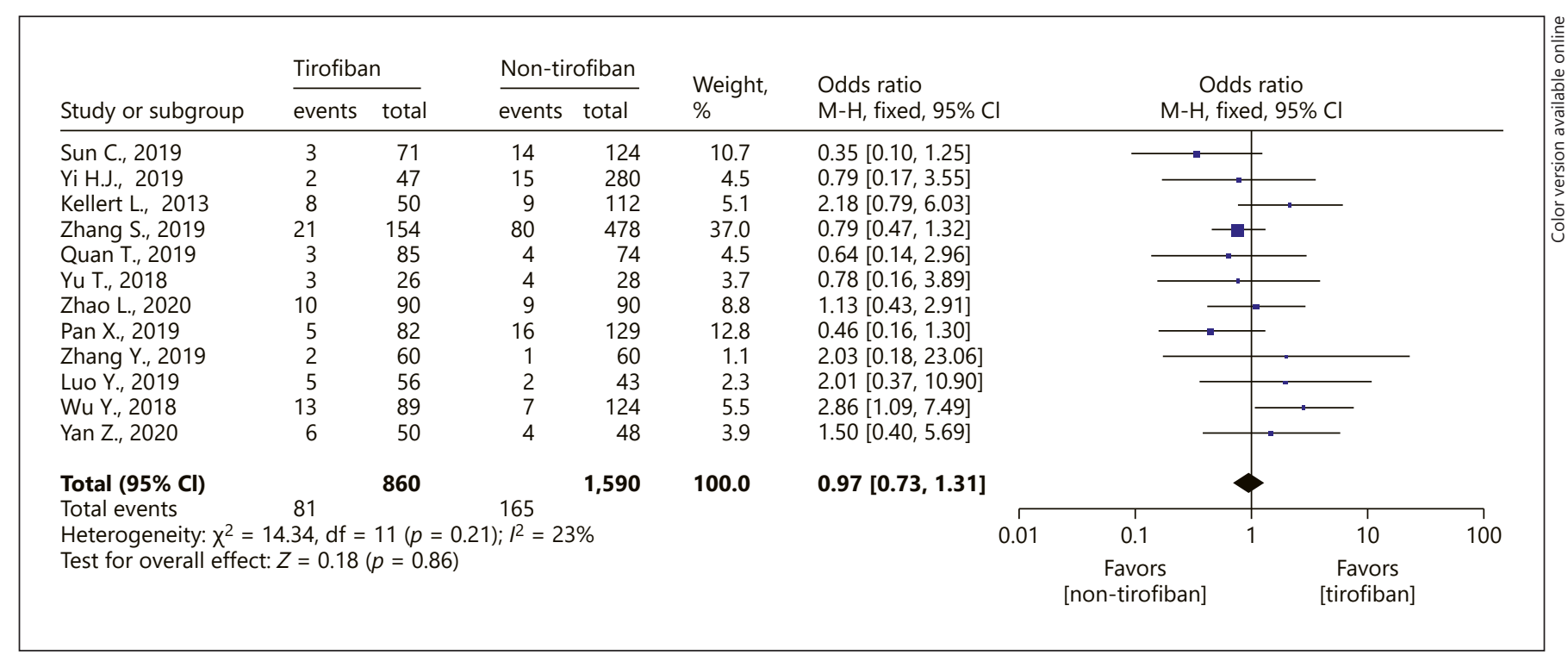

Fig. 6. Forest plot and meta-analysis of the risk of sICH. sICH, symptomatic intracerebral hemorrhage.

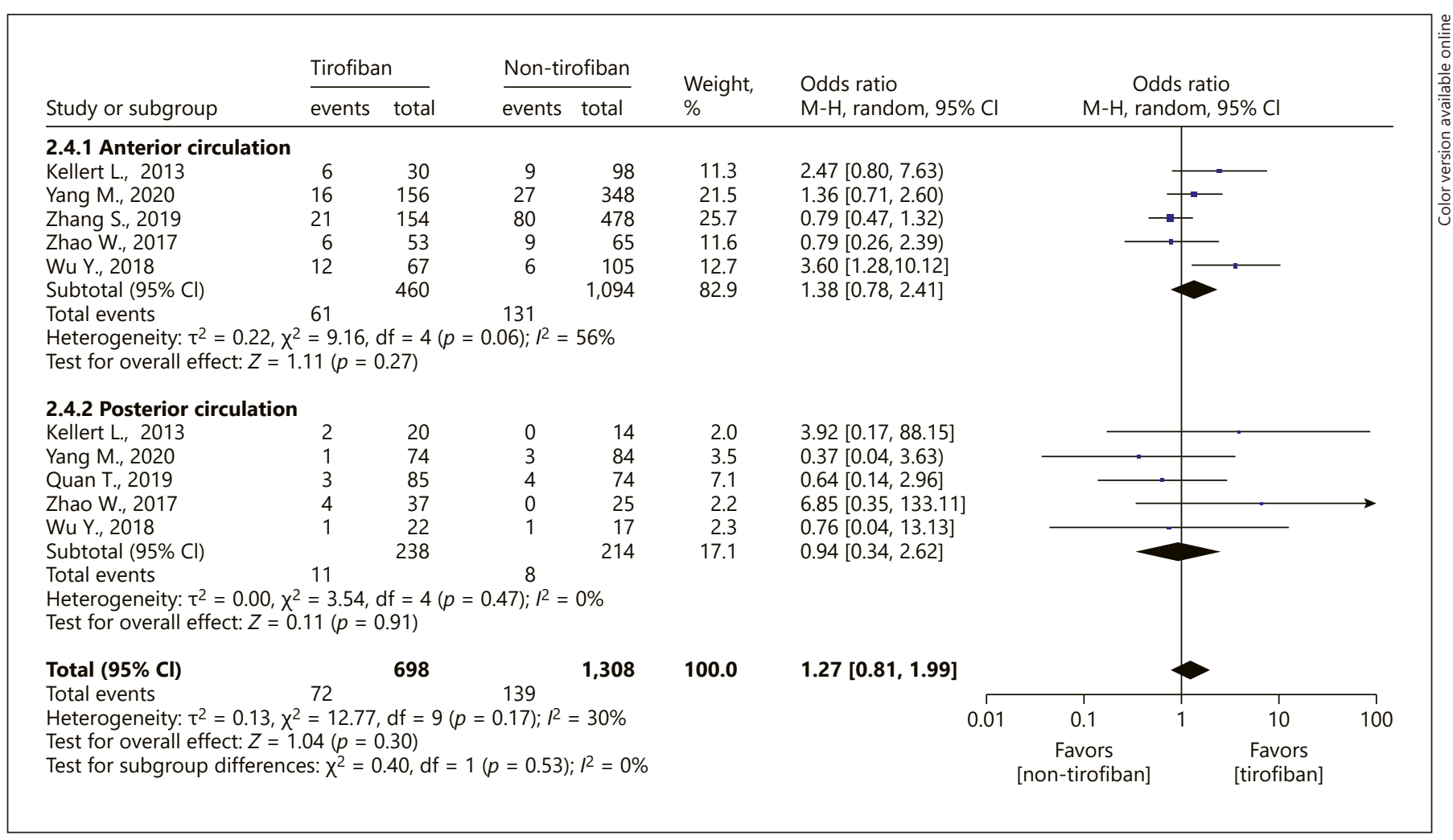

Fig. 7. Forest plot and meta-analysis of subgroup analysis of sICH in the anterior and posterior circulation. sICH, symptomatic intracerebral hemorrhage. 


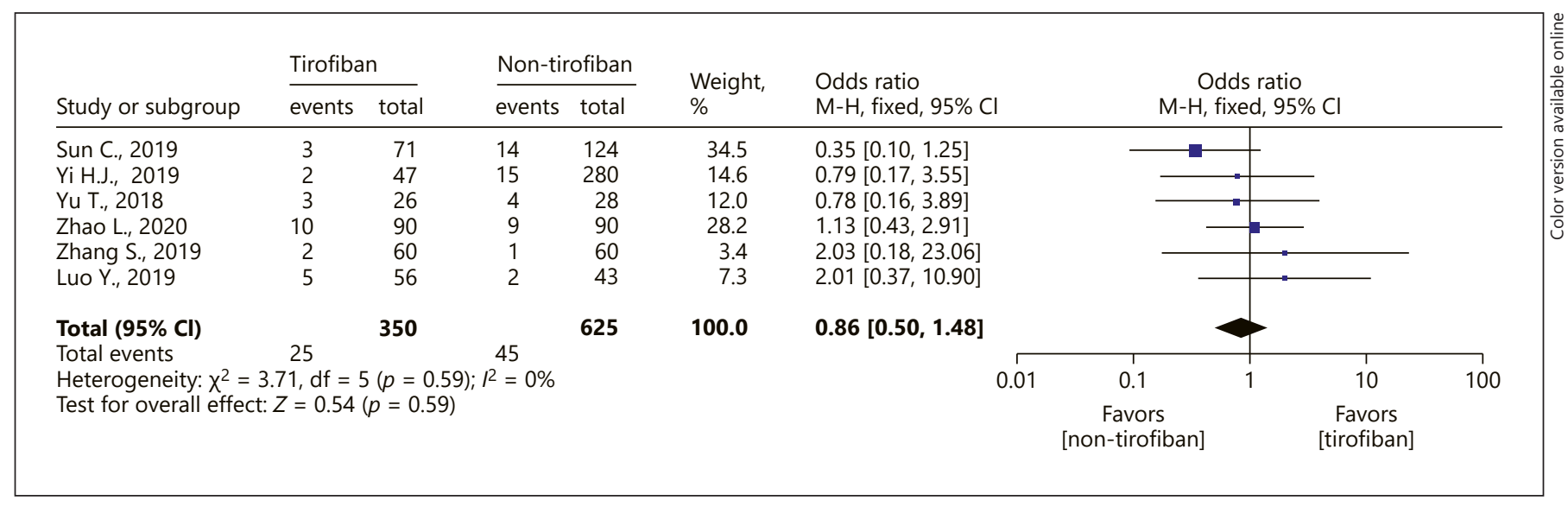

Fig. 8. Forest plot and meta-analysis of the risk of sICH in retriever stent subgroup. sICH, symptomatic intracerebral hemorrhage.

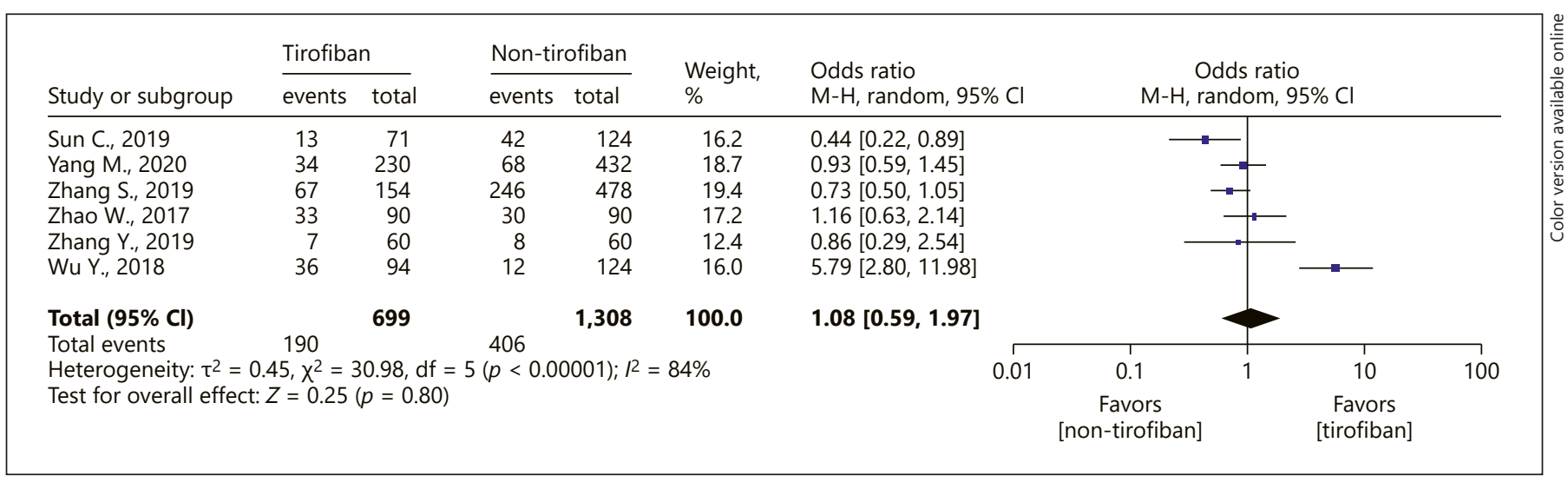

Fig. 9. Forest plot and meta-analysis of the risk of ICH. ICH, intracerebral hemorrhage.

might come from the article reported by $\mathrm{Wu}$ et al. [17], while this study was of relatively high quality. After excluding Wu et al.'s [17] article, heterogeneity decreased significantly ( $p=0.30, I^{2}=18 \%$ ), but no significant differences between the 2 groups have been found.

Thirteen studies [16, 17, 19-29] reported the mortality at 3-month in 3,077 patients with AIS with moderate heterogeneity $\left(I^{2}=42 \%, p=0.05\right)$. The tirofiban administration was associated with lower mortality; 200 of 1,080 patients died in the tirofiban group compared with 445 of 1,997 patients that died in the non-tirofiban group $(\mathrm{OR}=$ $0.75,95 \%$ CI $[0.62,0.91], p=0.003$; shown in Fig. 10).

\section{The Publication Biases}

In our meta-analysis, we used Begg's tests to evaluate the effect of publication bias. The funnel plots were sym- metrical. There was no obvious evidence of publication bias as revealed by Begg's funnel plots.

\section{Discussion}

The specific aim of the meta-analysis was to assess the safety and efficacy of tirofiban administration in EVT of AIS patients. A total of 14 articles involving 3,291 patients were enrolled. Our pooled analysis indicates that tirofiban could improve the 3-month functional outcome of AIS patients. For patients treated with retriever stent in EVT, the incidence of 3-month mRS 0-2 was significantly higher in patients with tirofiban compared to patients without tirofiban. Besides, tirofiban is associated with a higher postoperative recanalization rate. In terms of safe- 


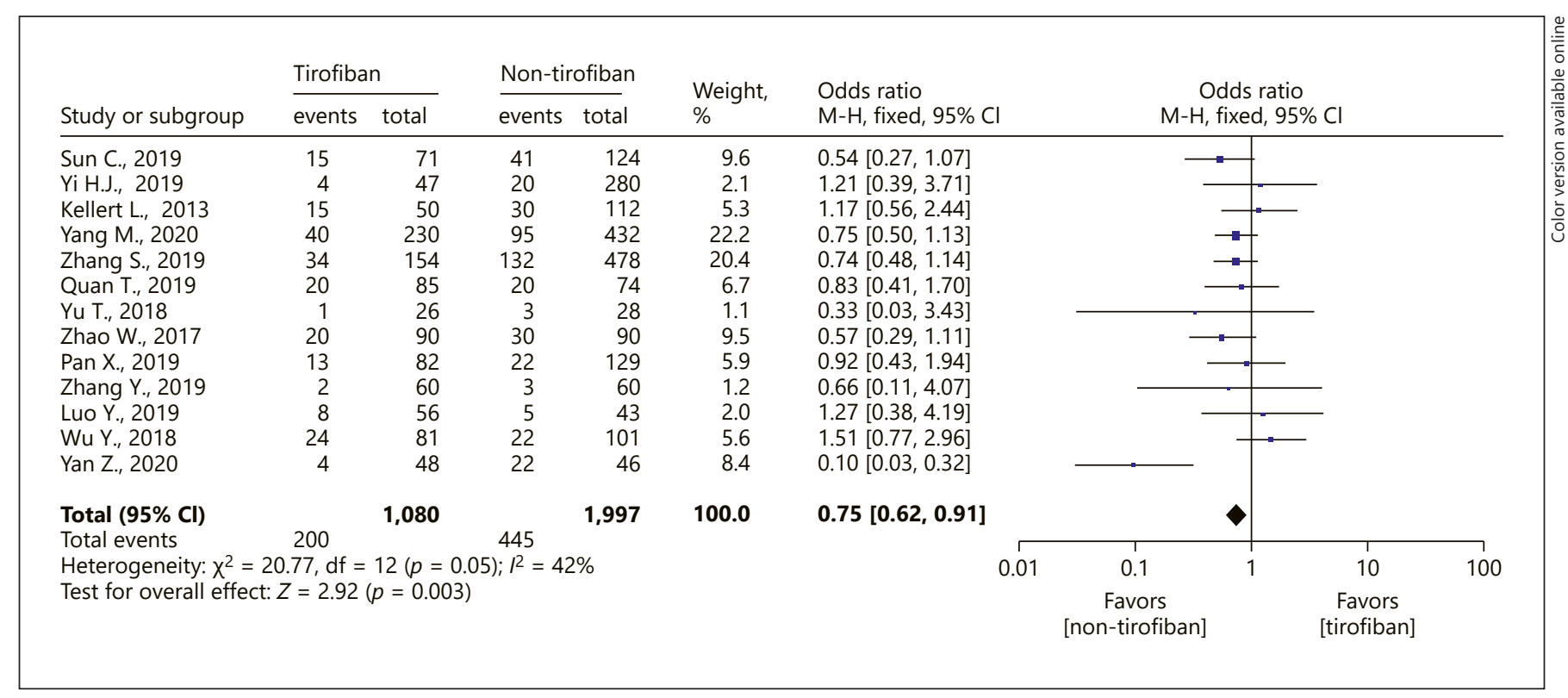

Fig. 10. Forest plot and meta-analysis of mortality at 3 months.

ty, our meta-analysis shows that tirofiban does not seem to increase the risk of sICH and ICH either in the anterior or posterior circulation. Also, tirofiban reduced the 3-month mortality of AIS patients when administered in EVT.

GP IIb/IIIa receptor antagonist has been widely used in the percutaneous coronary intervention in patients with acute myocardial infarction to prevent platelet aggregation and thrombosis by blocking fibrinogen-binding receptors reversibly $[31,32]$. Whereas due to the risk of hemorrhagic transformation, its application in stroke is limited [33]. Compared with abciximab and eptifibatide, tirofiban has a lower molecular weight, shorter halflife, and no immunogenicity, and its application in cerebrovascular diseases has been gradually accepted in recent years. However, the use of tirofiban in EVT of ischemic stroke remains controversial. Some studies [17, 19] showed that tirofiban increased the risk of hemorrhage and resulted in poor outcomes. By contrast, other studies $[16,34-36]$ reported that tirofiban could improve functional outcomes and postoperative recanalization rates without increasing hemorrhagic complications. Our review results are consistent with a previous meta-analysis of the safety of tirofiban in AIS patients not undergoing EVT [37]. The discrepancy between our results and that of Kellert et al. [19] and Wu et al. [17] may be due to a larger dose of tirofiban (e.g., $18 \mathrm{mg}$ for a $60-\mathrm{kg}$ adult) or a significantly higher proportion of subjects with atrial fibrillation in their study. Wu et al.'s [17] novel finding is that tirofiban may have a dose-dependent effect on ICH. Nevertheless, there is no consensus on the dosage of tirofiban in AIS, and large-scale tests are needed.

In addition to inhibiting platelet aggregation, tirofiban also inhibits inflammatory response and reduces reperfusion injury by inhibiting the expression of inflammatory factors, which may be another reason for improving the functional outcomes of AIS patients [38]. Thrombectomy is usually able to achieve recanalization of large blood vessels, but during this process, many broken small emboli may occlude the distal small blood vessels. Intraoperative application of tirofiban could effectively dissolve small embolus, reduce the occurrence of small vessel occlusion, and improve the long-term prognosis. We speculate that the dissolution of small embolus may be the key for tirofiban to improve the outcomes of patients treated with stent thrombectomy, which is consistent with the opinion proposed by Seo et al. [35].

In the absence of enough data, a meta-analysis was not conducted for in-stent thrombosis. Of the 14 studies analyzing tirofiban, only 1 study [27] reported stent thrombosis. They reported that the incidence of stent thrombosis was not statistically different in the 2 groups ( $0 / 60$ vs. $1 / 60, p=0.179$ ). This result may be due to small sample size or short follow-up interval ( 3 months). Besides, 2 trials assessed the application of tirofiban by stroke etiologies in AIS. Zhang et al. [26] reported that, compared with 
patients with cardioembolism, stroke patients of the large artery atherosclerosis subtype who treated with tirofiban were associated with low odds of death. And Sun et al. [24] also found that in patients with large artery atherosclerosis stroke, tirofiban seems to lead to favorable functional outcome, but cardioembolic stroke patients tended to have unfavorable prognosis. The inconsistency may be attributed to the actual structure of the thrombus, that is, the red thrombus often seen in cardioembolic-induced occlusion is enriched in RBCs, while the white thrombus consisting of platelets is usually seen in large artery atherosclerosis-induced occlusion [39]. Therefore, patients with atherosclerotic occlusion of large arteries may benefit from tirofiban.

\section{Limitations}

Some limitations need to be highlighted. First, the enrolled studies in the study were non-randomized controlled trials with a low level of evidence. Second, there is heterogeneity between studies, and subgroup or sensitivity analysis is not satisfactory for the problem. Third, the overall sample size of this study is small and may have an impact on the results. Fourth, the majority of the population included in this study is from Asia; the results may apply to Asian patients but not to Western patients.

\section{Conclusions}

The application of tirofiban in EVT of AIS may improve functional outcomes and reduce mortality at 3 months. Besides, tirofiban does not seem to increase the risk of $\mathrm{sICH}$ and $\mathrm{ICH}$, either in the anterior or PCS. More large-sample, multicenter, prospective randomized controlled studies are needed for further confirmation.

\section{Acknowledgements}

There are no acknowledgements to declare.

\section{Statement of Ethics}

Ethical approval was not required because it is a systematic review of previous published studies. The research was conducted ethically in accordance with the World Medical Association Declaration of Helsinki.

\section{Conflict of Interest Statement}

The authors have no conflicts of interest to declare.

\section{Funding Sources}

The authors did not receive any funding.

\section{Author Contributions}

L.T. participated in the design of the study, collected and analyzed the data, and drafted and revised the manuscript. Q.Y. analyzed the data, interpreted the results, and performed the statistical analysis and revised the manuscript. X.T. designed the study, supervised the study inclusion and data extraction, and revised the manuscript.

\section{References}

1 Benjamin EJ, Virani SS, Callaway CW, Chamberlain AM, Chang AR, Cheng S, et al. Heart disease and stroke statistics-2018 update: a report from the American Heart Association. Circulation. 2018 Mar 20;137(12):e67-e492.

2 Powers WJ, Rabinstein AA, Ackerson T, Adeoye OM, Bambakidis NC, Becker K, et al. Guidelines for the early management of patients with acute ischemic stroke: 2019 update to the 2018 guidelines for the early management of acute ischemic stroke: a guideline for healthcare professionals from the American Heart Association/American Stroke Association. Stroke. 2019 Dec;50(12):e344-418.

3 Berkhemer OA, Fransen PS, Beumer D, van den Berg LA, Lingsma HF, Yoo AJ, et al. A randomized trial of intraarterial treatment for acute ischemic stroke. N Engl J Med. 2015 Jan 1;372(1):11-20.
4 Goyal M, Demchuk AM, Menon BK, Eesa M, Rempel JL, Thornton J, et al. Randomized assessment of rapid endovascular treatment of ischemic stroke. N Engl J Med. 2015 Mar 12; 372(11):1019-30.

5 Jovin TG, Chamorro A, Cobo E, de Miquel MA, Molina CA, Rovira A, et al. Thrombectomy within 8 hours after symptom onset in ischemic stroke. N Engl J Med. 2015 Jun 11; 372(24):2296-306.

6 Saver JL, Goyal M, Bonafe A, Diener HC, Levy EI, Pereira VM, et al. Stent-retriever thrombectomy after intravenous t-PA vs. t-PA alone in stroke. N Engl J Med. 2015 Jun 11;372(24): 2285-95.
7 Bracard S, Ducrocq X, Mas JL, Soudant M, Oppenheim C, Moulin T, et al. Mechanical thrombectomy after intravenous alteplase versus alteplase alone after stroke (THRACE): a randomised controlled trial. Lancet Neurol. 2016 Oct;15(11):1138-47.

8 Campbell BC, Mitchell PJ, Kleinig TJ, Dewey HM, Churilov L, Yassi N, et al. Endovascular therapy for ischemic stroke with perfusionimaging selection. N Engl J Med. 2015 Mar 12; 372(11):1009-18.

9 Muir KW, Ford GA, Messow CM, Ford I, Murray A, Clifton A, et al. Endovascular therapy for acute ischaemic stroke: the pragmatic ischaemic stroke thrombectomy evaluation (PISTE) randomised, controlled trial. J Neurol Neurosurg Psychiatry. 2017 Jan;88(1):3844. 
10 Heo JH, Lee KY, Kim SH, Kim DI. Immediate reocclusion following a successful thrombolysis in acute stroke: a pilot study. Neurology. 2003 May 27;60(10):1684-7.

11 Power S, Matouk C, Casaubon LK, Silver FL, Krings T, Mikulis DJ, et al. Vessel wall magnetic resonance imaging in acute ischemic stroke: effects of embolism and mechanical thrombectomy on the arterial wall. Stroke. 2014 Aug;45(8):2330-4.

12 Marto JP, Strambo D, Hajdu SD, Eskandari A, Nannoni S, Sirimarco G, et al. Twenty-fourhour reocclusion after successful mechanical thrombectomy: associated factors and longterm prognosis. Stroke. 2019 Oct;50(10): 2960-3.

13 Platelet Receptor Inhibition in Ischemic Syndrome Management in Patients Limited by Unstable Signs and Symptoms (PRISMPLUS) Study Investigators. Inhibition of the platelet glycoprotein IIb/IIIa receptor with tirofiban in unstable angina and non-Q-wave myocardial infarction. N Engl J Med. 1998 May 21;338(21):1488-97.

14 Siebler M, Hennerici MG, Schneider D, von Reutern GM, Seitz RJ, Röther J, et al. Safety of tirofiban in acute ischemic stroke: the SaTIS trial. Stroke. 2011;42(9):2388-92.

15 Li W, Lin L, Zhang M, Wu Y, Liu C, Li X, et al. Safety and preliminary efficacy of early tirofiban treatment after alteplase in acute ischemic stroke patients. Stroke. 2016 Oct;47(10): 2649-51.

16 Zhao W, Che R, Shang S, Wu C, Li C, Wu L, et al. Low-dose Tirofiban improves functional outcome in acute ischemic stroke patients treated with endovascular thrombectomy. Stroke. 2017 Dec;48(12):3289-94.

17 Wu Y, Yin C, Yang J, Jiang L, Parsons MW, Lin L. Endovascular thrombectomy: tirofiban increases bleeding risk in acute stroke patients. Stroke. 2018 Nov;49(11):2783-5.

18 Hacke W, Kaste M, Bluhmki E, Brozman M, Dávalos A, Guidetti D, et al. Thrombolysis with alteplase 3 to 4.5 hours after acute ischemic stroke. N Engl J Med. 2008 Sep 25; 359(13):1317-29.

19 Kellert L, Hametner C, Rohde S, Bendszus M, Hacke W, Ringleb P, et al. Endovascular stroke therapy: tirofiban is associated with risk of fatal intracerebral hemorrhage and poor outcome. Stroke. 2013 May;44(5):14535 .
20 Yu T, Lin Y, Jin A, Zhang P, Zhou X, Fang M, et al. Safety and efficiency of low dose intraarterial tirofiban in mechanical thrombectomy during acute ischemic stroke. Curr Neurovasc Res. 2018;15(2):145-50.

21 Luo Y, Yang Y, Xie Y, Yuan Z, Li X, Li J. Therapeutic effect of pre-operative tirofiban on patients with acute ischemic stroke with mechanical thrombectomy within 6-24 hours. Interv Neuroradiol. 2019 Dec;25(6):705-9.

22 Pan X, Zheng D, Zheng Y, Chan PWL, Lin Y, Zou J, et al. Safety and efficacy of tirofiban combined with endovascular treatment in acute ischaemic stroke. Eur J Neurol. 2019 Aug;26(8):1105-10.

23 Quan T, Hou H, Xue W, Yu G, Ma H, Sun J, et al. Endovascular treatment of acute intracranial vertebrobasilar artery occlusion: a multicenter retrospective observational study. Neuroradiology. 2019;61(12):1477-84.

24 Sun C, Li X, Zhao Z, Chen X, Huang C, Li X, et al. Safety and efficacy of tirofiban combined with mechanical thrombectomy depend on ischemic stroke etiology. Front Neurol. 2019 Oct 29;10:1100.

25 Yi HJ, Sung JH, Lee DH. Safety and efficacy of intra-arterial tirofiban injection during mechanical thrombectomy for large artery occlusion. Curr Neurovasc Res. 2019;16(5): 416-24.

26 Zhang S, Hao Y, Tian X, Zi W, Wang H, Yang $\mathrm{D}$, et al. Safety of intra-arterial tirofiban administration in ischemic stroke patients after unsuccessful mechanical thrombectomy. J Vasc Interv Radiol. 2019 Feb;30(2):141-e1.

27 Zhang Y, Zhang QQ, Fu C, Wang L, Zhang GQ, Cao PW, et al. Clinical efficacy of tirofiban combined with a Solitaire stent in treating acute ischemic stroke. Braz J Med Biol Res. 2019;52(10):e8396.

28 Yan Z, Shi Z, Wang Y, Zhang C, Cao J, Ding $\mathrm{C}$, et al. Efficacy and safety of low-dose tirofiban for acute intracranial atherosclerotic stenosis related occlusion with residual stenosis after endovascular treatment. Journal Stroke Cerebrovasc Dis. 2020 Apr;29(4):104619.

29 Yang M, Huo X, Gao F, Wang A, Ma N, Shi $\mathrm{H}$, et al. Low-dose rescue tirofiban in mechanical thrombectomy for acute cerebral largeartery occlusion. Eur J Neurol. 2020;27(6): 1056-61.
30 Zhao L, Jian Y, Li T, Wang H, Lei Z, Sun M, et al. The safety and efficiency of tirofiban in acute ischemic stroke patients treated with mechanical thrombectomy: a Multicenter Retrospective Cohort Study. Biochem Res Int. 2020 May 1;2020:5656173.

31 Lefkovits J, Plow EF, Topol EJ. Platelet glycoprotein IIb/IIIa receptors in cardiovascular medicine. N Engl J Med. 1995 Jun 8;332(23): 1553-9.

32 Schwarz M, Meade G, Stoll P, Ylanne J, Bassler N, Chen YC, et al. Conformation-specific blockade of the integrin GPIIb/IIIa: a novel antiplatelet strategy that selectively targets activated platelets. Circ Res. 2006 Jul 7; 99(1):25-33.

33 Yang M, Huo X, Miao Z, Wang Y. Platelet glycoprotein IIb/IIIa receptor inhibitor tirofiban in acute ischemic stroke. Drugs. 2019 Apr; 79(5):515-29.

34 Mangiafico S, Cellerini M, Nencini P, Gensini $G$, Inzitari D. Intravenous tirofiban with intra-arterial urokinase and mechanical thrombolysis in stroke: preliminary experience in 11 cases. Stroke. 2005 Oct;36(10):2154-8.

35 Seo JH, Jeong HW, Kim ST, Kim EG. Adjuvant tirofiban injection through deployed Solitaire stent as a rescue technique after failed mechanical thrombectomy in acute stroke. Neurointervention. 2015 Feb;10(1): 22-7.

36 Goh DH, Jin SC, Jeong HW, Ha SY. Mechanical Solitaire thrombectomy with low-dose booster tirofiban injection. Neurointervention. 2016 Sep;11(2):114-9.

37 Zhou J, Gao Y, Ma QL. Safety and efficacy of tirofiban in acute ischemic stroke patients not receiving endovascular treatment: a systematic review and meta-analysis. Eur Rev Med Pharmacol Sci. 2020;24(3):1492-503.

38 Lee JS, Hong JM, Lee KS, Suh HI, Choi JW, Kim SY. Primary stent retrieval for acute intracranial large artery occlusion due to atherosclerotic disease. J Stroke. 2016;18(1):96101

39 Wang XG, Zhang LQ, Liao XL, Pan YS, Shi YZ, Wang CJ, et al. Unfavorable outcome of thrombolysis in chinese patients with cardioembolic stroke: a Prospective Cohort Study. CNS Neurosci Ther. 2015 Aug;21(8): 657-61. 